\title{
Tecnología de gestión de los efluentes líquidos del camal municipal de Chachapoyas, Amazonas, 2017
}

\section{Technology for the management of liquid effluents from the municipal camal, Chachapoyas, Amazonas, 2017}

\author{
Sonia Edith Sánchez Díaz
}

\section{RESUMEN}

Esta investigación tuvo como objetivo elaborar una Tecnología de Gestión del camal municipal de Chachapoyas, Amazonas, 2017. Se realizaron trabajos en el camal en diferentes días de la semana durante un mes; verificando y clasificando tipo de animal, número de sacrificios, masa de animal sacrificado, masa de desecho líquido; con la información de SENASA se calculó la producción anual. En la recolección de muestras y mediciones colaboraron un promedio de 25 personas. El número mensual de sacrificios de ganado vacuno, porcino y ovino es 327,353 y 28 animales respectivamente, dando un total anual de 8492 ganados, con un peso promedio para vacuno y $90,7 \mathrm{~kg}$ para porcino. El porcentaje de líquido residual de cada ganado fue de $16 \%$ del vacuno y $8 \%$ del porcino. El flujo promedio de desechos líquidos fue 904,2 m3 mensuales y $10850.8 \mathrm{~m} 3$ anual. En la evaluación de la buena práctica de manufactura hay deficiencias en maquinaria, procedimientos, registros sanitarios, registro de información, etc. Los residuos líquidos totales son eliminados directamente en la red de alcantarillado y éste al río Sonche, contaminado este curso de agua y ocasionando pérdidas económicas pudiendo ser transformados en producto alimenticio para animales, lumbricultura y compostaje.

Palabras clave: Residuos líquidos, buena práctica de manufactura, camal municipal

\begin{abstract}
The objective of this research was to develop a management technology for the Chachapoyas - Amazonas municipal camal in 2017. Work was carried out in the camal on different days of the week for a month; verifying and classifying type of animal, number of sacrifices, mass of slaughtered animal, mass of liquid waste; with the information of SENASA the annual production was calculated. An average of 25 people collaborated in the collection of samples and measurements. The monthly number of sacrifices of cattle, pigs and sheep is 327, 353 and 28 animals respectively, giving an annual total of 8492 cattle, with an average weight of 296.7 for cattle and $90.7 \mathrm{~kg}$ for pigs. The percentage of residual liquid of each cattle was $16 \%$ of the cattle and $8 \%$ of the pig. The average flow of liquid waste was $904,2 \mathrm{~m} 3$ per month and $10850.8 \mathrm{~m} 3$ per year. In the evaluation of good manufacturing practice there are deficiencies in machinery, procedures, health records, information registration, etc. The total liquid waste is eliminated directly in the sewerage network and this to the Sonche River, contaminated this watercourse and causing economic losses, being able to be transformed into a foodstuff for animals, lumbriculture and composting.
\end{abstract}

Keywords: soil analysis, soil analysis parameters, soil quality. 


\section{INTRODUCCIÓN}

En la actualidad, en el camal municipal de Chachapoyas se vienen sacrificando un promedio 22 animales diariamente entre ganado vacuno, porcino y ovino; los desechos sólidos y los efluentes líquidos son eliminados al medio ambiente sin ningún control. En el caso de los efluentes líquidos son desechados a la red de desagüe de la ciudad que se dirige al río Sonche, sin criterio alguno de efecto ambiental de contaminación. El presente proyecto tiende a minimizar esta contaminación con el fin de proteger la biodiversidad de la cuenca del río Sonche, en marco al reglamento de ley de recursos hídricos, DS. $\mathrm{N}^{\circ}$ 001-2010-AG. y evitar generación de posibles enfermedades en la población, proliferación de vectores, asegurar un consumo de carnes de buena calidad y con las características necesarias para el consumo humano y un trabajo en el camal donde se realice la mejora continua en todas sus áreas. Esta es la importancia de la elaboración de una tecnología de gestión de los efluentes líquidos del camal municipal de Chachapoyas, ya que en la actualidad es una necesidad económica y una necesidad de higiene pública, debido que la principal fuente de contaminación se encuentra en las aguas residuales de los mataderos; dentro de los principales contaminantes tenemos a la heces y orina, sangre, pelusa, lavaza, residuos de carne y grasas en los canales, alimentos no digeridos por los intestinos, tripas de animales sacrificados.

Las ventajas para la población y el medio ambiente sería menor impacto ambiental, aprovechamiento total de subproductos, fomento del empleo, disminución o control del costo de concentrado para animales. Para lo cual se está caracterizando y conceptualizando los efluentes líquidos del camal municipal de Chachapoyas; se ha propuesto el aprovechamiento de estos efluentes y la determinación de los posibles beneficios a la población como posibles microempresas de tratamiento de los efluentes o centros de rendering autorizados, donde se puedan obtener productos como alimento de ganado, aves y peces; así como permitir la conservación de la biodiversidad con la propuesta de una planta de tratamiento del resto de los efluentes líquidos.

\section{MATERIAL Y MÉTODOS}

Objeto de estudio

Para los efluentes líquidos del camal municipal, se verificó y clasificó según tipo de animal sacrificado, número de sacrificios, masa del animal sacrificado.
Para posteriormente proponer una Tecnología de Gestión: Programa para mitigar, corregir, controlar y prevenir o eliminar los impactos negativos por la contaminación del agua; la mala disposición de los efluentes, la falta de capacitación, los riesgos y peligros a los que están expuestos los trabajadores y la población en general; y las actividades a desarrollar, así como las estrategias a utilizar, los costos que implican la implementación.

Diseño de la investigación

Diseño experimental: Diseño clásico. (Ostle 1973).

\section{Población, muestra y muestreo}

El universo está formado por los diferentes efluentes del $100 \%$ de animales sacrificados y del agua a utilizar durante el proceso desde la recepción hasta la salida de la carne limpia lista a expendio.

Esto se ha realizado en función al tipo, número y masa del animal sacrificado.

Se ha verificado la producción de efluentes líquidos en diferentes días de la semana durante un mes, y con la información alcanzado por SENASA, se realiza la producción anual.

\section{Fuentes de información}

La información fue obtenida y comparada de cuatro fuentes: Por los investigadores y colaboradores, la alcanzada por el director del camal municipal, de SENASA y EMUSAP.

\section{Métodos, técnicas, instrumentos y procedimientos}

En la universidad si se cuenta con un laboratorio de análisis de aguas, pero tiene un costo, y este proyecto no cuenta con presupuesto, no se ha realizado un análisis de las aguas residuales, como se tenía planteado inicialmente. En su lugar se ha realizado la recolección de todos los residuos líquidos y sólidos por días durante un mes y se ha verificado el gasto de agua durante ese periodo.

En el proceso de recolección de residuos se ha medido masa y/o volúmenes según sea el caso; para el mismo se ha elaborado una ficha técnica con los siguientes datos: sangre, líquido ruminal, piel, cachos, casco, sólido ruminal, grasas no comestibles, mermas (especificar) y otros.

La realización de la recolección de muestras y sus mediciones han colaborado un total de 25 personas en horario de trabajo del camal.

Una vez recolectada la información se procesó según registro de faenado durante un mes. Se ha registrado los residuos o desechos por animal sacrificado teniendo en consideración la masa de animal vivo, 
sacando un promedio en porcentaje de cada tipo de desechos de cada animal. El promedio de porcentaje de residuos, se multiplica por el promedio de animales por mes y luego por año, luego se le adiciona el gasto total de agua por me y anual utilizado, obteniendo el volumen total de líquidos residuales provenientes del proceso de faenado del camal municipal, por mes y por año durante el periodo enero-diciembre 2017.

\section{RESULTADOS}

Tabla 1. Promedio de sacrificios mensual de ganado vacuno, ovino y porcino en el año 2017.

\begin{tabular}{|l|l|l|l|}
\hline \multirow{2}{*}{$\begin{array}{l}\text { Tipo de } \\
\text { ganado }\end{array}$} & $\begin{array}{l}\text { Promedio del } \\
\text { número de } \\
\text { sacrificios }\end{array}$ & \multirow{2}{*}{$\begin{array}{l}\text { Porcentaje } \\
\text { anual \% }\end{array}$} \\
\cline { 2 - 3 } & Mes & Año & \\
\hline VACUNO & 327 & 3921 & 46,2 \\
\hline PORCINO & 353 & 4235 & 49,9 \\
\hline OVINO & 28 & 336 & 4,0 \\
\hline TOTAL & 708 & 8492 & 100 \\
\hline
\end{tabular}

Tabla 2. Peso promedio de animal

\begin{tabular}{|l|l|}
\hline Tipo de ganado & $\begin{array}{l}\text { Peso promedio } \\
\text { del animal (Kg) }\end{array}$ \\
\hline VACUNO & 296,7 \\
\hline PORCINO & 90,7 \\
\hline
\end{tabular}

Tabla 3: Porcentaje promedio de desechos del ganado vacuno respecto al peso del animal vivo.

\begin{tabular}{|l|l|l|}
\hline $\begin{array}{l}\text { Subproductos o } \\
\text { desechos del } \\
\text { matadero }\end{array}$ & $\begin{array}{l}\text { Porcen } \\
\text { taje } \\
\mathbf{( \% )}\end{array}$ & Observaciones \\
\hline SANGRE & 7 & $\begin{array}{l}\text { panza, cuajo, intestinos } \\
(9 \%)\end{array}$ \\
\hline $\begin{array}{l}\text { CONTENIDO } \\
\text { RUMINAL }\end{array}$ & 26 & \\
\hline $\begin{array}{l}\text { PIEL, CACHOS } \\
\text { Y CASCOS }\end{array}$ & 10 & $\begin{array}{l}\text { Fragmentos de tejidos } \\
\text { (desperdicios de } \\
\text { matanza) }\end{array}$ \\
\hline $\begin{array}{l}\text { SÓLIDO DEL } \\
\text { LIBRO }\end{array}$ & 3,5 & $\begin{array}{l}\text { Hígados enfermos, } \\
\text { ubres con mastitis, críos }\end{array}$ \\
\hline COMASAS NO & 2,5 & \\
\hline MERMAS & 3 & $0-12,5$ \\
\hline OTROS* & 52 & \multicolumn{2}{|l}{} \\
\hline TOTAL &
\end{tabular}


Tabla 4: Porcentaje promedio de desechos del ganado porcino respecto al peso del animal vivo.

\begin{tabular}{|l|l|l|}
\hline $\begin{array}{l}\text { Subproductos o } \\
\text { desechos del } \\
\text { matadero }\end{array}$ & $\begin{array}{l}\text { Porcen } \\
\text { taje } \\
\mathbf{( \% )}\end{array}$ & Observaciones \\
\hline SANGRE & $\mathbf{4}$ & \\
\hline $\begin{array}{l}\text { CONTENIDO } \\
\text { RUMINAL }\end{array}$ & 6 & $\begin{array}{l}\text { panza, cuajo, } \\
\text { intestinos (4\%) }\end{array}$ \\
\hline CASCOS & 10 & \\
\hline $\begin{array}{l}\text { GRASAS NO } \\
\text { COMESTIBLES }\end{array}$ & 2,5 & \\
\hline MERMAS & 3 & $\begin{array}{l}\text { Fragmentos de } \\
\text { tejidos (desperdicios } \\
\text { de matanza) }\end{array}$ \\
\hline OTROS* & $0-5,5$ & $\begin{array}{l}\text { Hígados enfermos, } \\
\text { ubres con mastitis, } \\
\text { críos }\end{array}$ \\
\hline TOTAL & 25 & \multicolumn{2}{|l}{} \\
\hline
\end{tabular}

Tabla 5. Promedio de efluentes líquidos mensual del ganado vacuno y porcino.

\begin{tabular}{|l|l|l|}
\hline & \multicolumn{2}{|l|}{ Tipo de ganado } \\
\cline { 2 - 3 } & Vacuno & Porcino \\
\hline \% respecto al peso del animal vivo & 16 & 8 \\
\hline Peso promedio del animal vivo $(\mathrm{Kg})$ & 296,7 & 90,7 \\
\hline Peso promedio por animal respecto al peso del animal vivo $(\mathrm{Kg})$ & 47,5 & 7,3 \\
\hline $\mathrm{N}^{\circ}$ mensual de animal sacrificados & 356 & 168 \\
\hline Peso promedio mensual de efluentes líquidos $(\mathrm{Kg})$ & 16900 & 1226.4 \\
\hline Total de peso de efluentes líquidos $(\mathrm{Kg})$ & 18126,4 \\
\hline
\end{tabular}

Tabla 5. Promedio de efluentes líquidos mensual del ganado vacuno y porcino.

\begin{tabular}{|l|l|l|}
\hline Tipos de desechos líquidos & Mes & Año \\
\hline Peso de efluentes líquidos $(\mathrm{Kg})$ & 18126,4 & 217516,8 \\
\hline $\begin{array}{l}\text { Peso de aguas residuales mensuales producto de lavados en diferentes áreas de } \\
\text { proceso } \mathrm{m}^{3}\end{array}$ & 735,4 & 8825,0 \\
\hline $\begin{array}{l}\text { Peso de aguas residuales mensuales producto de lavados en diferentes áreas de } \\
\text { proceso Kg }\end{array}$ & 735417,0 & 8825000,0 \\
\hline Peso Total en Kg & 753543,1 & 9042516,8 \\
\hline Volumen Total en $\mathrm{m}^{3}(\mathrm{DR} \approx 1)$ & 753,5 & 9042,5 \\
\hline
\end{tabular}




\section{DISCUSIÓN}

Los resultados son producto del trabajo realizado desde los meses de julio a diciembre del 2017 y la información alcanzada por las diversas instituciones como el camal municipal, SENASA, EMUSAP.

El resultado de la recopilación de producción de enero a diciembre del año 2017, se encuentran en la tabla 1, donde están indicados los promedios en número y porcentaje de los sacrificios mensual del ganado vacuno, porcino y ovino. El número de sacrificios total del año 2017 es de 3921,4235 y 336 ganados entre vacuno, porcino y ovino respectivamente, el mismo que se utilizó para el cálculo el promedio mensual.

En la tabla 2, se encuentra el peso promedio del ganado vacuno y porcino, siendo el peso de $296,7 \mathrm{Kg}$ del ganado vacuno y $90,7 \mathrm{Kg}$ del ganado porcino. Este promedio en proporción a la bibliografía es de peso bajo, ya que en algunas referencias el promedio es $1000 \mathrm{~kg}$ y $800 \mathrm{~kg}$ para machos y hembras para el ganado vacuno; y el promedio para el ganado porcino es de $200 \mathrm{~kg}$. (Camal Municipal de LambayequePrograma de Manejo y Adecuación Ambiental) (GUERRERO J.y RAMIREZ I.2004)

En las tablas 3 y 4, se aprecia el porcentaje promedio de los desechos del ganado vacuno y porcino respecto al peso $(\mathrm{kg})$ del animal vivo, indicando que los desechos son sangre, contenido ruminal; piel, cachos y cascos; sólidos del libro; grasas no comestibles, fragmentos de desperdicios, hígados, ubres enfermos y críos. Estos promedios concuerdan en parte con los promedios obtenidos. Pero se debe recalcar que el porcentaje de sacrificios con hígados enfermos es elevado $58,5 \%$ al $90 \%$ en algunos días. Debido a que no existe control, ni tratamiento preventivo del animal vivo.

El promedio de los efluentes líquidos mensual del ganado vacuno y porcino, es de $18109,4 \mathrm{Kg}$ en el mes, se aprecia en la tabla 5.

En la tabla 6, es el resultado de la recopilación de la masa $(\mathrm{kg})$ y el volumen $\left(\mathrm{m}^{3}\right)$ total de los desechos líquidos por mes y por año que son eliminados directamente a la red de alcantarillado. Este promedio es de los efluentes líquidos de los sacrificios y el agua utilizado para el faenado. Esta masa y volumen es de $753526,4 \mathrm{~kg}$ y $904,2 \mathrm{~m}^{3}$ respectivamente promedio por mes. Asimismo la masa y el volumen es de $9042316,8 \mathrm{~kg}$ y $10850,8 \mathrm{~m}^{3}$ respectivamente promedio por año.

De manera General, en la evaluación de la buena práctica de manufactura del camal se aprecia muchas deficiencias en cuanto a maquinaria obsoleta, procedimiento inadecuado, sin control óptimo sanitario, sin registro de informaciones, con mucho descuido por parte de la municipalidad, pudiendo ocasional daños en la salud de la población chachapoyana.

\section{CONCLUSIONES}

El funcionamiento del Camal Municipal de Chachapoyas es de manera regular en todo el año, los 12 meses, 6 días a la semana.

La producción mensual de residuos líquidos (efluentes y aguas residuales) es de 904,2 $\mathrm{m}^{3}$ siendo la producción anual de $10850,8 \mathrm{~m}^{3}$.

Los residuos líquidos, sin tratamiento alguno, son eliminados directamente a la red de alcantarillado y estos al río Sonche, incrementado la contaminación de este curso de agua, entorpeciendo la vida acuática y degradando las corrientes de aguas abajo ya son utilizados por otros pueblos o actividades (criaderos de truchas).

La evaluación de la buena práctica de manufactura del camal indica muchas deficiencias en cuanto a maquinaria obsoleta, procedimiento inadecuado, sin control óptimo sanitario, sin registro de informaciones, sin capacitación del personal, con mucho descuido por parte de la municipalidad. Por tanto, según tabla 7 de anexos que a la municipalidad corregir y mejorar todos los aspectos de calificación hasta 2, para luego aplicar una mejora continua y posteriormente un Six sigma.

\section{REFERENCIAS}

Bogado A. (2010). Tratamiento de efluentes de matadero. Colombia.

C. Baird. (2001). Química Ambiental, Ed. Reverté. Capítulo 9

D.C. Harris. (2000). Análisis Químico Cuantitativo $2^{\mathrm{a}}$ ed., Ed. Reverte. Capítulos 14 al 18.

Escuela Organización Industrial. (2008). Contaminación de las aguas. Vertidos de mataderos e industrias cárnicas. Sevilla.

Gobierno regional de Lambayeque. Gerencia regional de Recursos Naturales y Gestión Ambiental. Camal municipal. Programa de Manejo y Adecuación Ambiental.

Guerrero J. y Ramirez I. (2004) Manejo ambiental de Residuos en mataderos de pequeños 
municipios. Scientia Et Technica, vol X, núm. 26. Universidad Tecnológica de Pereira. Colombia.

Hernández, Roberto y col. (2004). Metodología de la investigación. 3era. Edición. Editorial McGraw-Hill Interamericana. México. 689 pp.

Hómez M. (2012) Aspectos Técnicos para el aprovechamiento de residuos Orgánicos Generados en mataderos. ACODAL. Universidad de la Salle - Colombia.

Mongomery, Douglas. (1973). Diseño y Análisis de experimentos. Tercera edición. Grupo editorial Iberoamericana. México.

Muñoz D. (2005) Sistema de Tratamiento de aguas residuales de mataderos para una población menor 2000 habitantes. Universidad del cauca.

Ostle, Bernard. (1973). Estadística Aplicada. Editorial Limusa. Wiley S.A. México.

Peña A. (2016). Los mataderos y el medio mbiente. CEPIS.

Quille G. y Donaires T. (2013). Revista Investigaciones Altoandinas. UN del Altiplano. Vol. 15. $\mathrm{N}^{\circ}$ 01. Puno.

Quispe C. y Zambrano W. 1998. Manual de Análisis Químicos Medioambientales. $1^{\mathrm{a}}$ edición.

Reglamento Sanitario del Faenado de Animales de Abasto. D.S. $\mathrm{N}^{\circ}$ 015-2012-AG.

Salas G. Condorhuamán C. (2008) Tratamiento de las aguas residuyales de un centro de beneficio o matadero de ganado. Facultad de Química. UNMSM.

http://www.fao.org/docrep/004/T0566S/T0566 S14.htm. Ingresado 10 de febrero 2017.

http://siteresources.worldbank.org/INTUSWM/ Resources/Spanish_LivestockMarch06.p df. Ingresado 10 de febrero del 2017.

http://www.fao.org/docrep/004/T0566S/T0566 S14.htm Ingre-sado 10 de febrero del 2017.

Vojvodic, R. (2002). Estudio analítico y Sistémico de la Realidad del Departamento de Amazonas. $1^{\mathrm{a}}$ edic. Ed. Centro de Altos Estudios Nacionales. CAEN. AmazonasPerú. 